\title{
A short proof for the number of permutations containing pattern 321 exactly once
}

\author{
Alexander Burstein \\ Department of Mathematics \\ Howard University \\ Washington, DC 20059 USA \\ aburstein@howard.edu
}

Submitted: May 30, 2011; Accepted: Aug 23, 2011; Published: Sep 2, 2011

Mathematics Subject Classification: 05A05, 05A15

\begin{abstract}
We give a short proof for J. Noonan's result on the number of permutations containing pattern 321 exactly once.
\end{abstract}

In this paper, we give a short proof for the result of Noonan [3] that the number of permutations of length $n$ containing exactly one occurence of pattern 321 is $\frac{3}{n}\left(\begin{array}{c}2 n \\ n-3\end{array}\right)$. (To be precise, Noonan considered permutations avoiding patterns 123, but taking the reversal of those, i.e. reading them right-to-left, we get an additional nice property.) This fact was later re-proved by Noonan and Zeilberger [4] using generating functions.

A pattern is an equivalence class of sequences under order isomorphism. Two sequences $\tau_{1}$ and $\tau_{2}$ over totally ordered alphabets are order-isomorphic if, for any pair of positions $i$ and $j$, we have $\tau_{1}(i) \square \tau_{1}(j)$ if and only if $\tau_{2}(i) \square \tau_{2}(j)$, where $\square$ is any one of $<,=,>$. We say that a sequence $\sigma$ contains a pattern $\tau$ if $\sigma$ has a subsequence order-isomorphic to $\tau$, otherwise we say that $\sigma$ avoids $\tau$.

Example 1 The sequence $\sigma=3614725$ contains pattern $\tau=321$ since $\sigma$ has a subsequence 642 order-isomorphic to 321.

Notation 2 The set of permutations in $S_{n}$ (i.e. of length $n$ ) avoiding pattern $\tau$ is denoted $S_{n}(\tau)$. The set of permutations in $S_{n}$ containing pattern $\tau$ exactly $r$ times is denoted $S_{n}(\tau ; r)$.

In fact, in Example 1, 642 is the only occurrence of 321 in $\sigma$. In 1995, Noonan [3] enumerated permutations of length $n$ containing exactly one occurrence of pattern 321 .

Theorem 3 (Noonan) $\left|S_{n}(321 ; 1)\right|=\frac{3}{n}\left(\begin{array}{c}2 n \\ n-3\end{array}\right)$. 


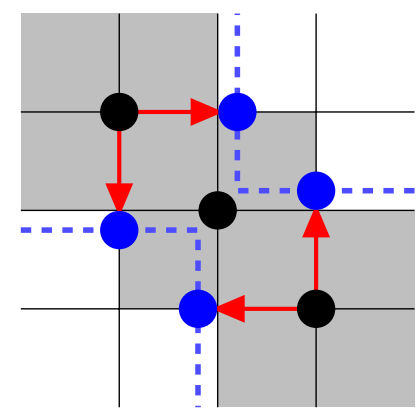

Figure 1: An injection $f: S_{n}(321 ; 1) \rightarrow S_{n+2}(321)$. The " 3 " and " 1 " in the unique occurrence of 321 are each replaced by two new points (in blue).

The proofs in $[3,4]$ enumerated a more general set of objects with additional restrictions and an auxiliary parameter. However, it was not apparent from those proofs why the above result was so simple and compact. We will use the block decomposition method of Mansour and Vainshtein [2] to make this much more apparent.

Proof Let $\pi \in S_{n}$ contain exactly one occurrence of pattern 321. Let $(c, b, a), c>b>a$, be the unique occurrence of 321 in $\pi$ at positions $(j, k, \ell), j<k<\ell$. Note that for $i<k, i \neq j$, we must have $\pi(i)<b$, or else $\pi$ will contain more than one occurrence of 321. Likewise, for $i>k, i \neq \ell$, we must have $\pi(i)>b$. Thus it is easy to see (for example, by exchanging values $c$ and $a$ at positions $j$ and $\ell$ ) that we must have $k=b$, so that $b$ is a fixed point of $\pi$. (This is the additional nice property we obtain by considering pattern 321 instead of 123.) Moreover, this implies that $j<k=b<c$ and $\ell>k=b>a$.

Define a map $f: S_{n}(321 ; 1) \rightarrow S_{n+2}(321)$ as follows. Let $\pi=\pi_{1} c \pi_{2} b \pi_{3} a \pi_{4}$. We will replace each of the points $(j, c)$ and $(\ell, a)$ on the permutation diagram of $\pi$ with two points as follows.

Let $0<\epsilon<1$ and let $\hat{\pi}$ be a permutation of the set $\{1,2, \ldots, b-1, b-\epsilon, b, b+\epsilon, b+$ $1, b+2, \ldots, n\}$ obtained by replacing the point $(j, c)$ with $(j, b-\epsilon)$ and $(b+\epsilon, c)$ and the point $(\ell, a)$ with $(b-\epsilon, a)$ and $(\ell, b+\epsilon)$ on the permutation diagram of $\pi$. In other words, let

$$
\hat{\pi}=\pi_{1}(b-\epsilon) \pi_{2} a b c \pi_{3}(b+\epsilon) \pi_{4}
$$

in one-line notation. Then $\pi^{\prime}=\pi_{1}(b-\epsilon) \pi_{2} a$ is a permutation of $\{1,2, \ldots, b-1, b-\epsilon\}$, $\pi^{\prime \prime}=c \pi_{3}(b+\epsilon) \pi_{4}$ is a permutation of $\{b+\epsilon, b+1, b+2, \ldots, n\}, \hat{\pi}=\pi^{\prime} b \pi^{\prime \prime}$, and $\pi^{\prime}<b<\pi$ (i.e. every entry in $\pi^{\prime}$ is less than $b$ and every entry in $\pi^{\prime \prime}$ is greater than $b$ ). Finally, define $f(\pi)$ to be the unique permutation in $S_{n+2}$ order-isomorphic to $\hat{\pi}$.

For instance, in Example 1, we obtain $f(3614725)=341258967$.

It is easy to see now that both $\pi^{\prime}$ and $\pi^{\prime \prime}$ avoid 321, so $f(\pi)$ avoids 321 as well, i.e. $f(\pi) \in S_{n+2}(321)$. Moreover, $f$ is clearly an injection. Furthermore, the only restrictions on $\pi^{\prime}$ (resp. $\pi^{\prime \prime}$ ) other than 321-avoidance are that it is nonempty and its greatest (resp. least) element is distinct from its rightmost (resp. leftmost) element. Thus, $f$ is a bijection from $S_{n}(321)$ onto its image, i.e. the subset of $S_{n+2}(321)$ that consists of permutations 
$\left(\rho^{\prime}, b+1, \rho^{\prime \prime}\right)$ such that $1 \leq b \leq n, \rho^{\prime}$ is a 321 -avoiding permutation of $\{1, \ldots, b\}$ that does not end on $b$, and $\rho^{\prime \prime}$ is a 321-avoiding permutation of $\{b+2, \ldots, n+2\}$ that does not start with $b+2$.

It is known that $\left|S_{n}(321)\right|=C_{n}$, the $n$th Catalan number (see [1]). Let $C=C(x)=$ $\sum_{n=0}^{\infty} C_{n} x^{n}$, then $C=1+x C^{2}$, so that $C=(1-\sqrt{1-4 x}) /(2 x)$. Therefore, the generating function for the number of nonempty 321-avoiding permutations whose greatest value is not their rightmost value (or whose least value is not their leftmost value) is

$$
C-1-x C=x C^{2}-x C=x C(C-1)=x C\left(x C^{2}\right)=x^{2} C^{3} .
$$

We leave it as an exercise to the reader to "bijectify" the above identity (e.g. map $\pi^{\prime}$ or $\pi^{\prime \prime}$ onto a disjoint union of 2 special points and 3 objects enumerated by Catalan numbers).

Therefore, the generating function for the number of permutations containing pattern 321 exactly once is

$$
x^{-2} \cdot\left(x^{2} C^{3}\right)^{2} \cdot x=x^{3} C^{6},
$$

and hence the number of such permutations is $\frac{3}{n}\left(\begin{array}{c}2 n \\ n-3\end{array}\right)$, as desired. The two factors of $x^{2} C^{3}$ correspond to $\pi^{\prime}$ and $\pi^{\prime \prime}$, the factor of $x^{-2}$ corresponds to the fact that $|\pi|-|f(\pi)|=$ $n-(n+2)=-2$, and the factor of $x$ corresponds to the fixed point $(b, b)$ of $\pi$.

It may be interesting to see if a similar strategy can be applied to give a nicer bijective proof for $\left|S_{n}(321 ; 2)\right|=\left(59 n^{2}+117 n+100\right) \frac{(2 n-2) !}{(n-4) !(n+5) !}$. It can also be easily modified to give the distribution for the statistic of the number of fixed points on $S_{n}(321 ; 1)$, since the map $f$ preserves the number of fixed points.

\section{References}

[1] D. E. Knuth. The Art of Computer Programming, vols. 1,3. Addison-Wesley Publishing, MA, 1968, 1973.

[2] T. Mansour, A. Vainshtein, Restricted permutations and Chebyshev polynomials, Sém. Lothar. Combin., B47c (2002), 17 pp.

[3] J. Noonan, The number of permutations containing exactly one increasing subsequence of length three, Discrete Math. 152 (1996), no. 1-3, 307-313.

[4] J. Noonan, D. Zeilberger, The enumeration of permutations with a prescribed number of "forbidden" patterns, Adv. Appl. Math. 17 (1996), no. 4, 381-407. 